\title{
The Structure of Coliphages
}

\author{
By D. E. BRADLEY \\ Department of Zoology, University of Edinburgh, Scotland
}

(Received 11 October 1962)

\begin{abstract}
SUMMARY
The detailed morphology of several new coliphages is compared with that of the more familiar types; the resulting information is considered for its taxonomic value. Some were examined at molecular level and new information on the tail structure of the ' $T$ even' phages is described. It is shown that the so-called tail fibres are, in fact, the remains of a network which surrounds the extended sheath. From this information it is possible to propose the changes in molecular packing accompanying sheath contraction.

A new phage with an octahedral head and contractile tail is described. It is also shown that phage T3 almost certainly has an octahedral head. Electron micrographs of $\phi \mathrm{R}$ (a $\phi \times 174$ type phage) show that it consists of two parts : an icosahedral protein shell, and morphological subunits attached to each apex.
\end{abstract}

\section{INTRODUCTION}

Bacteriophages specific to Escherichia coli include a number of different morphological types. These are mostly restricted to the $\mathbf{T}$ group and relatively few others have been described. In the present work, some new coliphages which have been isolated from sewage-contaminated water are illustrated; some of them are morphologically distinct from those in the $T$ group. There are three morphological types within the T group (Bradley \& Kay, 1960): the ' $T$ even' phages with contractile sheaths, T1 and T5 with long tails without contractile sheaths, and T3 and T7 with very short tails. The very small phages $\phi$ X 174 (Sinsheimer, 1959; Tromans \& Horne, 1961) and $\phi \mathrm{R}$ (Bradley, 1961; Kay, 1962; Kay \& Bradley, 1962) constitute another group. The phages described here add three more morphological types; each group is studied in some detail. In the cases of phages T2 and T4 the molecular changes in the tail which lead to nucleic acid injection can be deduced.

\section{METHODS}

Sources of bacteriophages used. The following kindly supplied the bacteriophages mentioned: Dr D. Kay (Sir William Dunn School of Pathology, University of Oxford), phages T2, T3, T5, $\phi$ R, 66t- and also Escherichia coli strains B and c2; Dr and Mrs K. G. Lark (Saint Louis University School of Medicine, U.S.A.) phages $\mathbf{T} 4$ and $\mathbf{T} 5$ and also $E$. coli strain $\mathbf{F}$.

Phage $\mathrm{C} 1$ was obtained by growing a typhoid phage $(\phi 1$, Fildes \& Kay, 1955) on Escherichia coli $\mathrm{c} 2$ and phage $\mathrm{F} 1$ from a second typhoid phage ( $\phi 2$, Bradley \& Kay, 1960) grown on $E$. coli F, both original phages being sent by Dr Kay.

Phage E 1 was isolated from a water sample from near Aldermaston, Berkshire, and phages $\alpha 1, \alpha 3, \beta 4$ and $\gamma 6$ from water samples (rivers, streams, reservoirs) taken 
within a 50-mile radius of Edinburgh. The host in all these cases was Escherichia coli $\mathbf{c 2}$, save for phage $\mathrm{E} 1$ which was grown on $\boldsymbol{E}$. coli $\mathrm{\text {B. }}$

Isolation, growth and purification of phages. Escherichia coli $\mathrm{c} 2$ was used to isolate phages from water samples. The following procedure was used. An overnight slope culture of $E$. coli $\mathrm{c} 2$ was suspended in $1 \mathrm{ml}$. water. One $\mathrm{ml}$. of the water sample which contained $0.1 \mathrm{M}$-ammonium acetate and thymol was then added to the bacterial suspension; $1 \mathrm{ml}$. of this mixture was plated out by the double agar layer method (Adams, 1950). After incubation, the plaques formed were picked and suspended in $0.1 \mathrm{M}$-ammonium acetate solution. Plaques were selected by their morphology, and the resulting phage suspension was further purified by plating and plaque picking. In general the phage count for water samples varied from $\mathbf{1}$ to $\mathbf{5 0}$ plaque-forming particles specific to $E$. coli $\mathrm{c} 2 / \mathrm{ml}$. water. Samples from sewagecontaminated rivers contained more phage particles than did samples from reservoirs or ponds.

Phages were grown in broth cultures (in the cases of the T phages and phage $\phi R$ a defined medium was used) or as follows. Sufficient phage particles were plated out by the double agar layer method to cause the lysis of all the host bacteria. Phages were eluted by pouring $2-3 \mathrm{ml}$. of $0.1 \mathrm{M}$-ammonium acetate onto the plate after incubation, and allowing it to stand for some hours. The decanted ammonium acetate solution usually contained about $10^{\text {11 }}$ particles from each plate. Phage suspensions were purified by several cycles of centrifugation. Ammonium acetate solution $(0 \cdot 1 \mathrm{M})$ was used as a suspending medium in most cases because phages are very stable in it. With phage $\phi \mathbf{R}$ a special purification procedure was used (Kay, 1962).

Electron microscope specimen preparation. The negative contrast method of Brenner \& Horne (1959a) was used throughout. The embedding chemicals were potassium phosphotungstate, sodium molybdate and uranyl acetate. Shadowing with platinum metal or platinum + carbon (Bradley, 1959) was also used.

\section{RESULTS \\ 'T even' type phages}

Plate 1 , fig. 1 shows phage $T 4$ which was suspended in $0.1 \mathrm{M}$-ammonium carbonate solution and embedded in sodium molybdate as a negative stain. What is best described as a collar can be clearly seen between the base of the head and the top of the tail sheath. In addition, two parallel lines join the base-plate of the tail and the collar outside the sheath. At two points (arrowed) the lines split into a network. The phage thus appears to possess an external structure surrounding the tail sheath. The nature of this assembly is illustrated where the negative staining material has caused the disruption of the sheath (Pl. 1, fig. 2). Here, the centre portion of the sheath is missing, but the top and bottom remain intact; two pairs of fibres join the portions. The appearance is consistent with the presence of six fibres, two of which are invisible being obscured by the tail core. This is a logical number since it is known that the base-like plate is hexagonal (Brenner \& Horne, 1959b), and one might expect a fibre to be attached at each corner. Plate 1, fig. 3 also shows clearly that the structure is fibrous in nature; it has been broken up and its appearance is remarkably like the often observed tail fibres of phage T2. From these three 
micrographs it can be seen that the sheath is surrounded by a fibrous network. As will become apparent, results with phage $\mathbf{T} 2$ show that the tail fibres are, in fact, the broken network.

The newly isolated phage $\alpha 1$, which is morphologically similar to phage $\mathbf{T} 4$, has some structure in the collar (Pl. 1, fig. 4), which is likely to possess sixfold radial symmetry like the rest of the tail. A typhoid phage $66 \mathrm{t}^{-}$(Pl. 1, fig. 5) also has a fibrous outer network and this and the collar are shown even more clearly and consistently than in phage $\mathbf{T} 4$.

Phage T2 is illustrated in Pl. 2, figs. 7-10. A few particles possessed a fibrous network (Pl. 2, figs. 7, 8), but no tail fibres. Others (Pl. 1, figs. 9, 10) had tail fibres, but no network. No obvious collar was found as in the other ' $T$ even' type phages.

Plate 2, fig. 10 shows a number of different components: two intact particles, kinked tail fibres $(f)$, a core and base-plate $(c)$, and a contracted sheath $(s)$. The latter has twelve distinct cross-striations with a spacing of about $33 \AA$. Similar periodicities were found on the contracted sheaths of phages T4 and $66 \mathrm{t}^{-}$(Pl. 3, fig. 17).

In a very few micrographs, the negative staining and preservation were sufficiently good to show the actual subunits of the extended sheath in phages $\mathrm{T} 2$ and $\mathrm{T} 4$. They are illustrated, together with a possible model, in Pl. 3, fig. 11. Their arrangement will be discussed below.

Phage E1. Plate 3, figs. 12 and 13 illustrate two intact particles of phage $\mathbf{E} 1$ with superimposed models of octahedra for comparison with the head shape. The tail sheaths are extended and exhibit a subunit structure. There are also about twenty-six cross-striations with a $45 \AA$ spacing. There is an indistinct base-plate. A phage with a contracted sheath is shown in Pl. 3, fig. 15. This has distinct parallel longitudinal striations in addition to rather indistinct cross-striations numbering 12 or 13. The shadowed phage (Pl. 3, fig. 16) clearly shows four tail fibres, only faintly visible in the negatively stained preparations.

Phage $C 1$. This phage has a large $(900 \AA)$ head and a long tail. The head shape is consistent with an octahedron and is shown in Pl. 4, fig. 18. The tail has crossstriations and a very fine point at the tip (Pl. 4, figs. 19, 22).

Phage F1. Phage F1 is very similar to phage $\mathrm{C} 1$ though it has not been possible to establish the head shape. The tail is different and is shown in the drawing in Pl. 4, fig. 21, which may be compared with the micrographs in Pl. 4, fig. 20.

Phage T 5. Platinum + carbon shadowing was used to establish the head shape. In Pl. 4, figs. 23 and 24 the shadows are cast by heads in orientations differing by about $60^{\circ}$ and are characteristic of an icosahedron. In Pl. 4, fig. 25, some facets of the head are visible and can be compared with the superimposed model of an icosahedron. Uranyl acetate preparations (Pl. 5, fig. 28), show marked cross-striations in the tail, caused presumably by the protein subunits of which it is built. There are forty striations in the whole length of the tail.

Phages $\beta 4$ and $\gamma 2$. Phage $\beta 4$ is shown in Pl. 5, fig. 29 and phage $\gamma 2$ in Pl. 5, fig. 30. They are similar in appearance and size (heads $600 \AA$, tails $1500 \AA$ ). The heads exhibit a hexagonal outline and the tails cross-striations. In the case of $\gamma 2$ there is a small group of fibres at the tail tip (arrowed) and the tails of $\beta 4$ have curled up, possibly due to the method of preparation. 
Phage T3. The appearance of this phage is familiar, but micrographs shown here (Pl. 5, figs. 31, 32) reveal two points of interest. In Pl. 5, fig. 31, a small fibre (arrowed) is attached to the tail, and both this micrograph and Pl. 5, fig. 32, strongly suggest that the head is an octahedron.

Small 'tailless' phages. Phages $\phi \mathbf{X} 174$ and $\phi R$ have been described in some detail, but one or two additional observations are mentioned here. Pl. 5, fig. 33, shows phage $\phi \mathbf{R}$ in phosphotungstate; the apical subunits of the icosahedron are clearly visible. In Pl. 5, fig. 34, an empty particle exhibits a continuous hexagonal outline and as in Pl. 5, fig. 33, some of the subunits are apparently divided into two parts. The icosahedron is lying on its twofold symmetry axis. Phage $\alpha 3$ is a morphologically similar phage isolated from a stream in Edinburgh, but is somewhat smaller (Pl. 5, fig. 35).

\section{DISCUSSION}

The structure of phages $T 2$ and $T 4$. In recent years since the detailed basic work of Brenner et al. (1959) there has been a good deal of discussion and speculation concerning the tail structure of these phages. Anderson (1960) observed a thin disk or collar on phage $\mathbf{T} 4$ between the base of the head and the top of the tail sheath. In addition to this, Daems, Van de Pol \& Cohen (1961) observed an outer jacket around the tail sheath and occasionally a helical structure superimposed on the well-known cross-striations. Apart from the fact that these components are definitely known to exist, no detailed information is available about them.

The nature of these features is considerably clarified by the micrographs shown here. The collar of phage $\mathbf{T} 4$ is particularly clear in $\mathrm{Pl}$. 1, fig. 1, and a micrograph of phage $\alpha 1$ (Pl. 1, fig. 4) suggests that its collar has a sixfold radial symmetry. This fits into the general symmetry pattern of the whole tail assembly. The collar is extremely delicate and has not yet been viewed end-on or isolated. Until this is done the most likely shape is considered to be a hexagonal plate resembling the base-plate (Brenner \& Horne, 1959b).

The so-called 'outer jacket' of Daems et al. (1961) is, in fact, a network or cage of fibres. It is difficult to discern the exact arrangement, but the micrograph of phage T4 (Pl. 1, fig. 1) suggests that the fibres branch some two-thirds of the way up the tail, each branch being attached to alternate apices of the collar.

The whole interrelated assembly of collar, fibrous network and base-plate is illustrated in the model in Pl. 1, fig. 6. While this may not be exactly correct, it is considered that the proposed structure will closely resemble the true arrangement of the components.

The micrographs of phage T2 provide complementary information. As observed by Daems et al. (1961) and originally shown by Brenner et al. (1959) phage T2 appears to possess no collar. However, Dr Horne (personal communication) and Dr Almeida (personal communication) have observed a distinct collar on their samples of this phage. Plate 2, figs. 7, 8, reveal a fibrous network, which is undoubtedly delicate and easily disrupted to form the so-called 'tail fibres'. The intact network appears to be attached to the head in the absence of a collar. In Pl. 2, fig. 9, the network has been partly disrupted and at the arrowed points the fibres appear to branch, as is consistent with the arrangement for phage $\mathbf{T} 4$. Plate 2, fig. $10 f$, shows the characteristically kinked 'tail fibres'; it seems most likely that the kink occurs 
where the fibres of the intact network branch, confirming that the 'tail fibres' are the broken network.

The coarse helical structure found on the phage T4 extended sheath by Daems et al. (1961) was not seen clearly on this phage but was occasionally visible on phage T2. It is shown by the arrows $C$ in Pl. 3, fig. $11 a$ and can be seen to be caused by a difference in density of some of the subunits, the arrangement being shown in the model (Pl. 3, fig. 11b). The arrowed subunits seem to be slightly bigger than the others, and since it is unlikely that the tail sheath contains two types of protein, they presumably represent the same subunits in a different orientation.

Plate 3, fig. 11 also suggests a reasonable arrangement for the subunits of the extended sheaths of phages T2 and T4, which appear to be similar. In Pl. 3, fig. $11 a$, the top row of four units is very clear, two more being out of sight. In the next two rows, sufficient are visible to allow the preparation of the model, which seems to agree reasonably well with the phage $\mathrm{T} 4$ sheath ( $\mathrm{Pl}$. 3, fig. 11c). Only the portions of the sheaths arrowed at $\boldsymbol{A}$ and $B$ show the subunit arrangement clearly. Elsewhere the structure is indistinct due to disorganization. This accounts for departures from the model which can be detected in some places. The arrangement shown is the most likely on the basis of the micrographs, but a single primary helix cannot be ruled out.

In the model, the subunits are related by screw symmetry and are in a series of annuli of sixfold radial symmetry. It is difficult to count the number of annuli exactly because in negatively stained preparations, the bottom few rows near the base-plate are often disorganized; however, there appear to be 24 or 25 , probably the former. The number is the same on all the T2-type phages. This gives a total of 144 or 150 subunits, the diameter of the visible profile being $30 \AA$ (compare Brenner et al. 1959, who suggested 200 subunits from molecular weight determinations).

In the case of the contracted sheath, all the T2-type phages mentioned here have twelve cross-striations (half the number in the extended sheath) with a spacing of about $33 \AA$. Brenner et al. (1959) showed that there was, in addition, a coarse helix.

From this information it is possible to make a plausible picture of the changes in molecular packing which accompany sheath contraction and the puncture of the cell wall. For the sheath to contract to half its length, the simplest mechanism would be to double the number of subunits from six to twelve per annulus. Thus, referring to the model (Pl. 3, fig. $11 b$ ) each ring of subunits would slip up into the interstices of the one above. If this were the case, one would expect axial striations to appear on the contracted sheath. In fact, the coarse helix found by Brenner et al. (1959) approaches axial striations, which have been found on at least three other phages with contractile tails (P1, Anderson, 1960; typhoid $\phi 2$, Bradley \& Kay, 1960; phage $\mathrm{E} 1$, present communication). In addition one would expect to find cross-striations slightly coarser than those in the extended sheath. Those observed were spaced $33 \AA$ as compared with $40 \AA$ for the extended sheath; they numbered, however, half those found on the extended sheath. Though these observations appear to be inconsistent, the presence of the correct number of striations on a contracted sheath is evidence in favour of the change in packing suggested. The likely explanation for the unexpected spacing difference is that the subunits are asymmetrical and probably rotate to some extent as well as changing their packing. This would also account for the fact that in the contracted sheath of the T2-type phages 
there is a coarse helix instead of axial striations. These results indicate that the tail sheath is much more than a collection of simply packed spherical subunits.

The function of the collar and fibrous network assembly is not known. One might expect it to have something to contribute towards the infective process. It is interesting to note that a collar is not always found on phage T2; the feature is doubtless more delicate than on other similar phages and liable to destruction during the preparation process.

The octahedral coliphages. Bradley \& Kay (1960) described a small phage with a contractile tail and a head which was probably an octahedron. Though it was a typhoid phage $(\phi 2)$, several apparently identical phages have been isolated from the Edinburgh area and Aldermaston on Escherichia coli C2. Their structure is important mainly because of the unusual head shape, but they also have an interesting tail assembly.

The initial appearance of $\phi 2$ heads in phosphotungstate suggested that they were octahedra; empty heads exhibited two characteristic superimposed triangles. It was found by using wire models, however, that a similar effect could be produced by an icosahedron when a number of its edges were rendered invisible, a possibility with negative staining. This is illustrated in Pl. 3, fig. 14. But in the case of the icosahedron (Pl. 3, fig. 14a) two of the sides of the triangle are kinked, while in the phage ( $\mathrm{Pl}$. 3, fig. 14b) and the octahedron (Pl. 3, fig. 14c) all the sides are straight. Further confirmation of the head shape was obtained with the morphologically identical phage $\mathbf{E} 1$, the actual faces of the octahedron being clearly visible in Pl. 3, figs. 12, 13. The heads of these phages therefore possess 4:3:2 symmetry.

The tail structure was studied on phage $\phi 2$ by Bradley $\&$ Kay (1960) but a few additional features are shown here. The extended sheaths in $\mathrm{Pl}$. 3, figs. 12, 13 have a structure which reflects the helical nature of the arrangement of the subunits, but it is not sufficiently clear to construct a model as with phages T2 and T4. Since the contracted sheaths have both longitudinal and transverse striations, it seems likely that the mechanism of sheath contraction is basically similar to that of the T2type phages.

At the end of the extended sheath there is a very small base plate, and four short tail fibres can be clearly seen attached to it in the shadowed phage (Pl. 3, fig. 16). This is consistent with a fourfold radial symmetry of the tail assembly, lying as it does on the fourfold axis of the octahedral head. Bearing in mind the fibrous network of the T2 type phages, the micrographs may not show the tail fibres in their true positions. In a very few cases (negatively stained) they seemed to be folded up the outside of the extended sheath.

Morphologically, this is a unique group of phages there being apparently no variants within it; it is common in sewage. Two Pseudomonas phages of very similar appearance have been found (Bradley, unpublished).

Large phages without contractile tails. Two phages are included in this group, $\mathrm{C} 1$ and F1. They are characterized by large heads and long tails. Phage $\mathrm{Cl}$ has an octahedral head. It has not been possible to establish the shape of the head of phage $\mathrm{F} 1$ because the heads usually collapse, but some micrographs also suggest an octahedron. The two phages are biologically different since they have different host ranges in strains of Escherichia coli, and the difference in tail structure confirms that they are taxonomically distinct. 
Smaller phages without contractile tails. Phage T 5 has been studied in some detail as a representative of a large group where the members are often similar morphologically. The evidence presented strongly suggests that phage T5 has an icosahedral head, but it is felt that an octahedron cannot be ruled out until better electron micrographs have been taken, perhaps by improved negative staining. The head shape is important in deducing the distribution of the protein subunits in the tail. It is possible by considering the radial and axial symmetry of the tail, its length, and also the size of the subunits, to build up a picture of the molecular structure.

The radial symmetry of the tail could not be observed directly on the few micrographs obtained of short lengths in section (Pl. 4, fig. $27 \mathrm{~s}$ ), but there is a possibility of deducing the symmetry from the geometrical form of the head. It is known that the T2-type phages possess a tail attached to their sixfold symmetry axis; its molecular structure is built up on a basis of sixfold radial symmetry. The four fibres of the octahedral phages, whose tail is attached to the fourfold symmetry axis, also indicate that it too possesses fourfold radial symmetry. Considering these precedents, it seems likely that other phage tails, including that of phage T5, also have the same radial symmetry as that of the axis to which they are attached. Phages, like many other types of virus, are clearly built on a strictly geometric basis and should therefore obey rules of symmetry. The tail of phage T5 is attached to a fivefold symmetry axis of the head, and, on the basis of the above remarks, should have fivefold radial symmetry. The axial arrangement of the subunits can be clearly seen in the tail (arrowed) in Pl. 5, fig. 28. They lie opposite one another, suggesting a series of annuli. The subunits appear to consist of a number of short tubes which can be seen end-on in the disorganized tail ( $\mathrm{Pl}$. 4, fig. 27, arrowed).

There are about 40 annuli per tail and with five subunits per annulus, based on the fivefold radial symmetry already discussed, there are some 200 subunits in all. The overall picture of the phage obtained by these results and deductions is illustrated in the model in Pl. 4, fig. 26, which is based on an icosahedron.

Phages $\beta 4$ and $\gamma 2$ represent a group resembling the SBL type of typhoid phage (Bradley \& Kay, 1960). They have fairly small heads (about $600 \AA$ ), long tails $(1500 \AA)$ and look generally similar to phage T5. Both of those shown here exhibit hexagonal outline of the head and cross-striations of the tail (PI. 5, figs. 29, 30). Their sizes are the same and also their general morphology. Since they differ somewhat in host range, a structural difference might be expected, and, after the study of phages $\mathrm{Cl}_{1}$ and F1, this might be in the appendage at the tail tip. Such an appendage has been found only in phage $\gamma 2$ and consists of a small group of fibres (Pl. 5, fig. 30, arrowed). In the case of phage $\beta 4$ the tails had curled up. This was noted with several similar phages and may well be due to the method of preparation. This will be the most difficult group to fit into any scheme of classification; morphologically they are very similar. Any differences will be very small and perhaps limited to tail appendages which are difficult to preserve and examine. The phages are very common.

Phages with very short tails. This morphological group is best represented by phage T3. It is mentioned here because a few examples have been found in water samples, but it is relatively uncommon. It is interesting to note that several similar morphological types have been found associated with other bacteria, for example 
Brucella abortus (Morgan, Kay \& Bradley, 1960) and also species of Pseudomonas (Bradley, unpublished).

The small 'tailless' phages. Phages $\phi \mathbf{R} 174$ (Sinsheimer, 1959; Tromans \& Horne, 1961) and $\phi R$ (Bradley, 1961; Kay, 1962; Kay \& Bradley, 1962) have been described in detail and their unique form is of interest. This morphological type is fairly common in sewage-contaminated waters and more than eight new isolates have recently been obtained (Bradley, 1962). One of them, phage $\alpha 3$, has been described above.

Phages $\phi \mathrm{X} 174$ and $\phi \mathrm{R}$ are known to consist of single-stranded DNA within an icosahedral protein coat. It seems very likely that the new isolates follow this pattern. Morphological subunits have been found at the apices of the icosahedron, and in the case of phage $\phi \mathbf{R}$ they have been resolved into two clearly defined portions (Bradley, 1961), shown in Pl. 5, figs. 33, 34 (arrowed). Their appearance is consistent with that of a tube on its side, but in $\mathrm{Pl}$. 5, fig. 34, where an empty particle is shown in another orientation, they still appear as definitely separated dots. This indicates that the subunits are really in the form of dimers and are not, in fact, short tubes. The protein container thus consists of two distinct parts, the continuous shell containing the DNA, and the apical subunits. The most important fact to discover now is whether these subunits play any part in the infective process, and if not, the mode of DNA injection used by the phage.

\section{Phage taxonomy}

It can be seen from the above results that there is a reasonable possibility of classifying phages on a basis of their morphology. Adams (1950) stated that there are three main criteria which should be applied in any system of phage classification: morphology, serological properties, host range characters. Let us consider first how purely morphological characters could be used to classify the coliphages studied here. The phages split into well defined groups: those with contractile tails (T2, E 1), those with non-contractile tails (F1, T5), and those with short tails or no tails $(\mathrm{T} 3, \phi \mathbf{R})$. This might seem to provide a reasonable basis for preliminary division, but within each group another important morphological character, namely, the shape of the head, can be different. In the first group there is the bipyramidal hexagonal prism of phage T2 and the octahedron of phage $\mathbf{E} 1$; in the second and third groups there are octahedra and icosahedra.

Such a grouping would clearly lead to confusion, and it is considered that it would be better to use a system of basic morphological types; some of these are listed in Table 1. It will be found that almost all the coliphages fit into one or other of these types. The exceptions are the F1 types and the smaller T 5 types; here, the tail structures which would have to be used to differentiate them have not yet been adequately examined. Such a system raises the question as to whether one phage is the same as another because it looks identical in spite of the fact that it may have a different host. Within group of phages specific to one species of bacterium this would raise no problem since the question of host range would only arise at strain level; it seems unreasonable to use strain differences in hosts to differentiate between what are effectively phage species. The greatest problem would be, for example, the case of phage T3; identical morphological types grow on Brucella and Pseudomonas organisms. 
Clearly, a purely morphological classification cannot be fully justified at present; serological characters must also be taken into account, and the difficulties of determining the serological relationships of a large number of phages are obvious. The use of a Linnæan type of classification would be premature, but it is felt that phages should be named in an informative manner, not merely by the bottle or batch number of the laboratory in which they were isolated, as this is inevitably completely meaningless on its own. In any original description of a phage the following could be mentioned: the reference number, the morphological type and the host genus or perhaps species. Thus the phage $\alpha \mathbf{1}$ described in this paper would be called $\alpha 1-\mathrm{T} 2$ (coli) indicating that it was similar in appearance with phage T2 and isolated on Escherichia coli. Such a system omits one extremely important piece of information, namely, the nature of the phage nucleic acid. This criterion provides an ideal way of dividing phages into large natural groups. Phage nucleic acid may be double-stranded DNA, single-stranded DNA, or RNA. A system of nomenclature of this kind seems essential if the present confusion is to be removed. It is hoped that the above suggestions will arouse the active interest of those concerned.

Table 1. Basic morphological types of coliphages

\begin{tabular}{|c|c|c|}
\hline \multirow{2}{*}{$\begin{array}{l}\text { Phage } \\
\text { morpho- } \\
\text { logical } \\
\text { type* }\end{array}$} & \multicolumn{2}{|c|}{ Characters } \\
\hline & Head shape & Tail type, etc. \\
\hline T2 & $\begin{array}{l}\text { Bipyramidal hexagonal } \\
\text { prism }\end{array}$ & Contractile sheath \\
\hline$\phi 2$ & Octahedron & Contractile sheath \\
\hline C1 & Octahedron. Large & $\begin{array}{l}\text { Non-contractile sheath; } \\
\text { pointed tip }\end{array}$ \\
\hline F 1 & Octahedron. Large & $\begin{array}{l}\text { Non-contractile sheath; } \\
\text { forked tip }\end{array}$ \\
\hline T5 & Icosahedron & $\begin{array}{l}\text { Non-contractile sheath; } \\
\text { tip obscure }\end{array}$ \\
\hline T3 & Octahedron & Very short \\
\hline$\phi \mathbf{X 1 7 4}$ & Icosahedron & Tailless, apical subunits \\
\hline
\end{tabular}

* The types mentioned are restricted to the coliphages and the names are those given when the phages were first described.

\section{Conclusion}

It is clear from the foregoing that the electron microscope can provide more information about phage structure than was originally expected. Straightforward observation has permitted something of the nature of the T $2 / \mathrm{T} 4$ injection mechanism to be understood at molecular level, and there seems no reason why similar success should not be obtained with other phages with contractile tails. The main problem of phage infection is, however, the manner in which the nucleic acid is transferred down the narrow tube of the tail and into the host and it is difficult to see just how the electron microscope can help in this respect. It does show, however, that almost every phage with a tail has some appendage at the tip and this presumably reacts with specific receptor sites on adsorption. The problem of the method of nucleic 
acid transfer used by phages of the $\phi \times 174$ type is unique since these do not seem to have conventional tails. It seems likely, however, that the apical subunits are in some way involved in the infective process.

The author is grateful to those listed above who provided phages and Miss $\mathbf{M}$. McCulloch and Mrs M. Moss for technical assistance. The author also wishes to thank Drs P. Wildy and D. Watson (Institute of Virology, University of Glasgow) for their valuable criticism and advice, and Professor M. M. Swann, F.R.S. (Head of the Zoology Department, University of Edinburgh) for his help and interest.

\section{REFERENCES}

Adams, M. H. (1950). Bacteriophages. New York and London: Interscience Publishers.

Anderson, T. F. (1960). On the fine structure of the temperate bacteriophages P1, P2 and P22. Proc. Eur. Reg. Conf. electron microscopy, Delft, 1960 2, 1008.

Bradley, D. E. (1959). High-resolution shadow-casting technique for the electron microscope using the simultaneous evaporation of platinum and carbon. Brit. J. appl. Phys. 10, 198.

Bradley, D. E. (1961). Negative staining of Bacteriophage $\phi R$ at various $p H$ values. Virology, 15, 203.

Bradley, D. E. (1962). Some new small bacteriophages ( $\phi$ X 174 type). Nature, Lond. 195, 622.

Bradley, D. E. \& KAY, D. (1960). The fine structure of bacteriophages. J. gen. Microbiol. 23, 553.

Brenner, S. \& Horne, R. W. (1959a). A negative staining method for high resolution electron microscopy of viruses. Biochim. biophys. Acta, 34, 103.

Brenner, S. \& Horne, R. W. (1959b). The structure of bacteriophage. The Times Science Review, London, Autumn, 1959.

Brenner, S., Streisinger, G., Horne, R. W., Champe, S. P., Barnett, L., Benzer, S. \& ReEs, M. W. (1959). Structural components of bacteriophage. J. mol. Biol. 1, 281.

Daems, W. Th., Van de Pol, J. H. \& Coren, J. A. (1961). Some remarks on the morphology of bacteriophage T4B. J. mol. Biol. 3, 225.

Findes, P. \& KAY, D. (1955). The rate of adsorption of bacteriophage by rough and smooth strains of Salmonella typhi. Brit. J. exp. Path. 36, 534.

KAY, D. (1962). The nucleic acid composition of bacteriophage $\phi R$. J. gen. Microbiol. 27, 201.

Kay, D. \& Braduey, D. E. (1962). The structure of bacteriophage $\phi$ R. J. gen. Microbiol. 27. 195.

Morgan, W. J. B., Kay, D. \& Bradley, D. E. (1960). Brucella bacteriophage. Nature Lond. 188, 74.

Sinshencer, R. L. (1959). Purification and properties of bacteriophage $\phi$ X174. J. mol. biol. $1,37$.

Tromans, W. J. \& Horne, R. W. (1961). The structure of bacteriophage $\phi$ X174. Virology, $15,1$.

\section{EXPLANATION OF PLATES 1-5}

Plate 1

Fig. 1. Phage $T 4$ in sodium molybdate, $\times \mathbf{3 3 3 , 0 0 0}$ (by courtesy of Academic Press Inc.).

Fig. 2. Phage $T 4$ in phosphotungstate, $\times 333,000$.

Fig. 3. Phage $T 4$ in sodium molybdate, $\times 383,000$.

Fig. 4. Phage $\alpha 1$ in phosphotungstate, $\times 333,000$.

Fig. 5. Phage 66t - in phosphotungstate, $\times 338,000$.

Fig. 6. Model of phage T4, $\times 300,000$. 

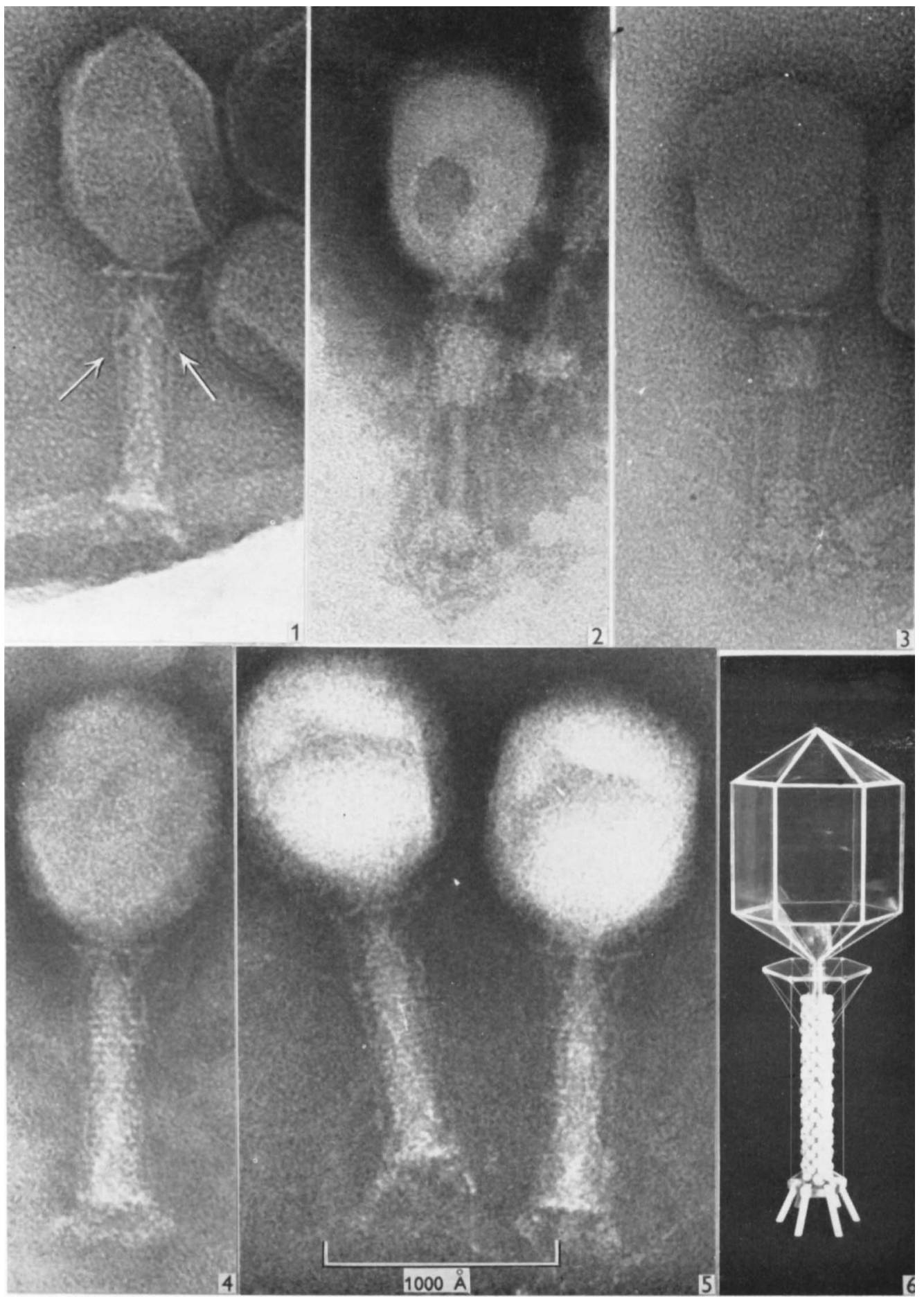

D. E, BRADLEY

(Facing p. 444) 
Journal of General Microbiology, Vol. 31, No. 3

Plate 2
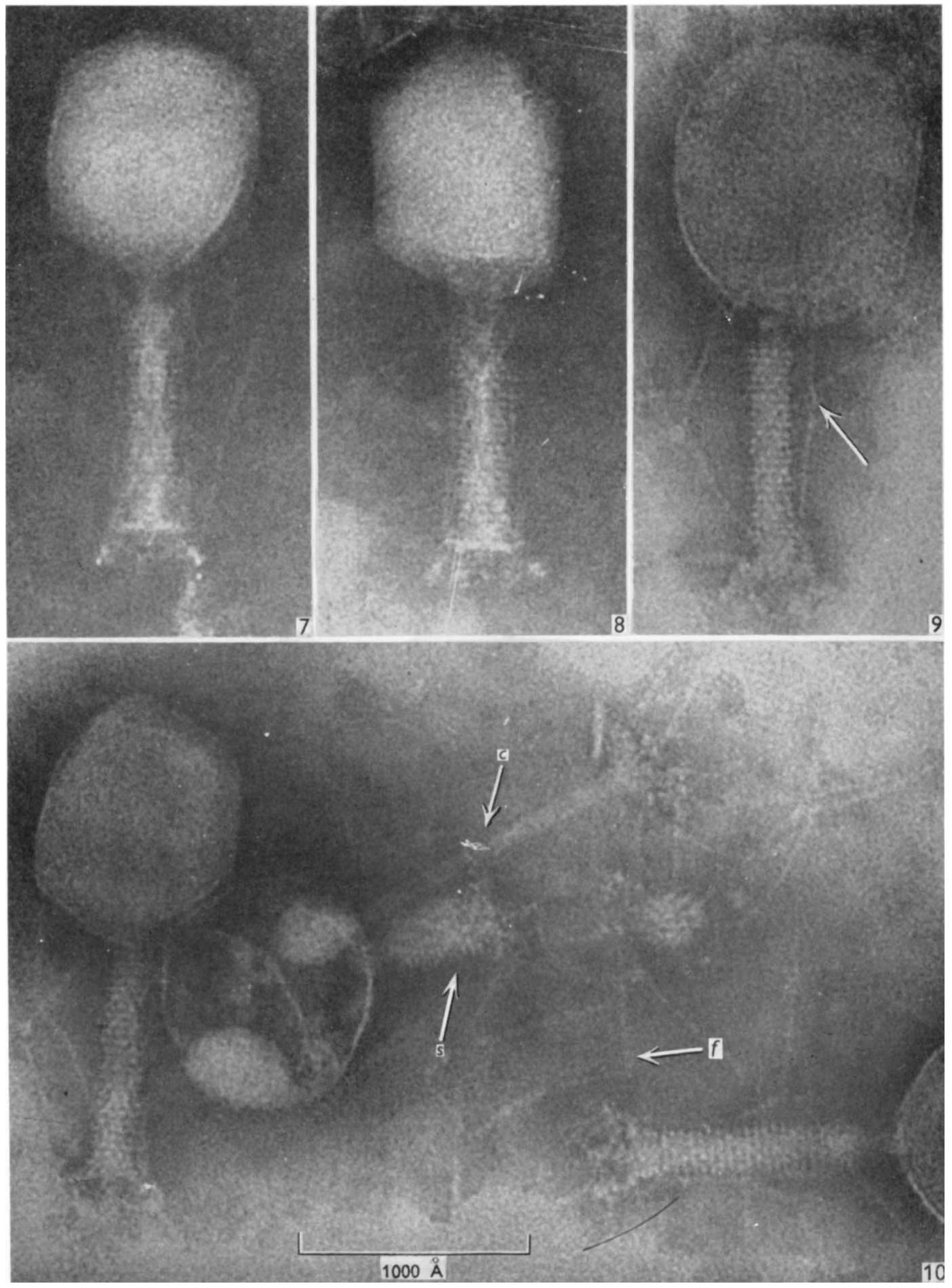

D. E. BRADLEY 
Journal of General Microbiology, Vol. 31, No. 3

Plate 3
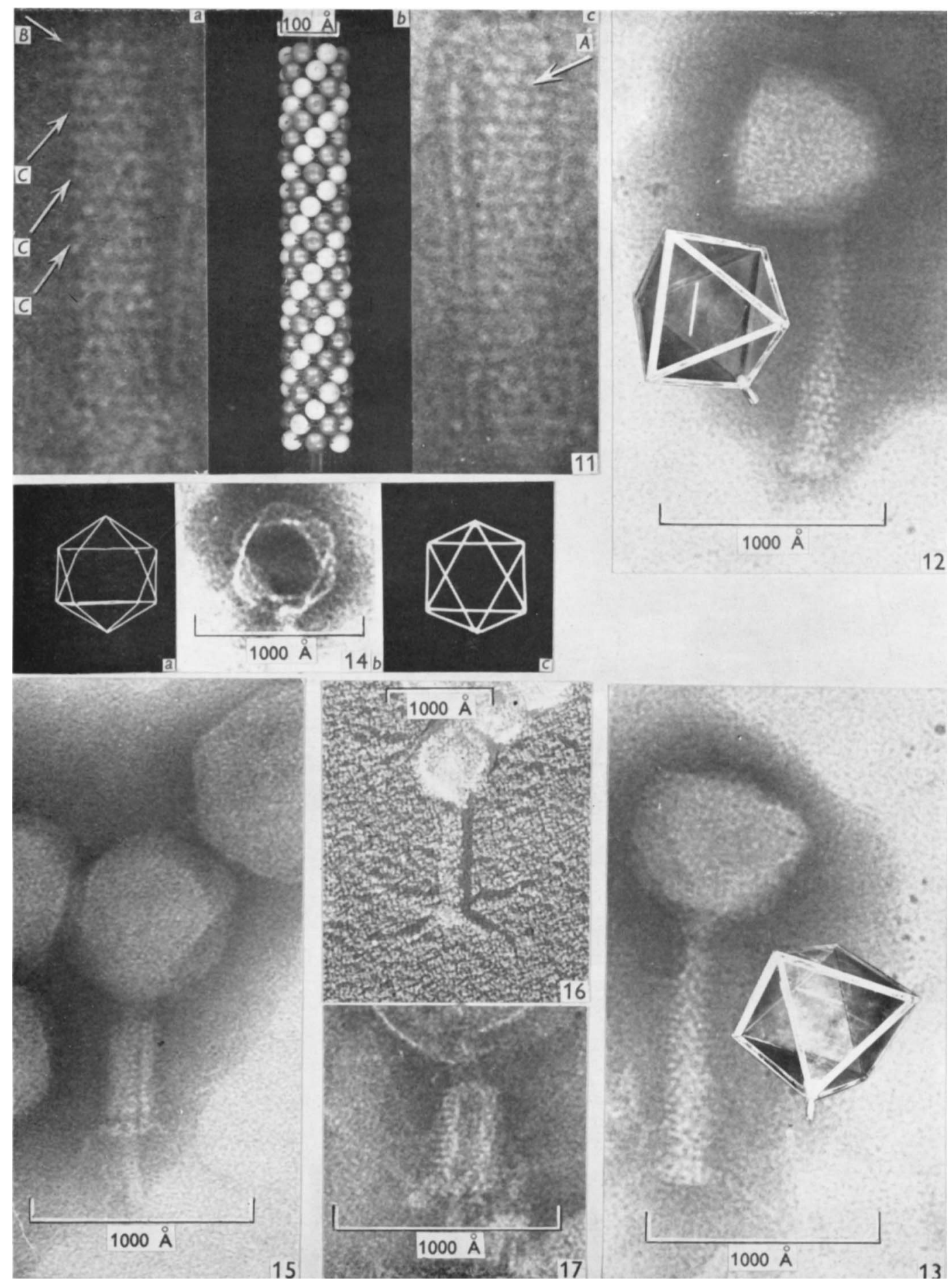

D. E. BRADLEY 
Journal of General Microbiology, Vol. 31, No. 3
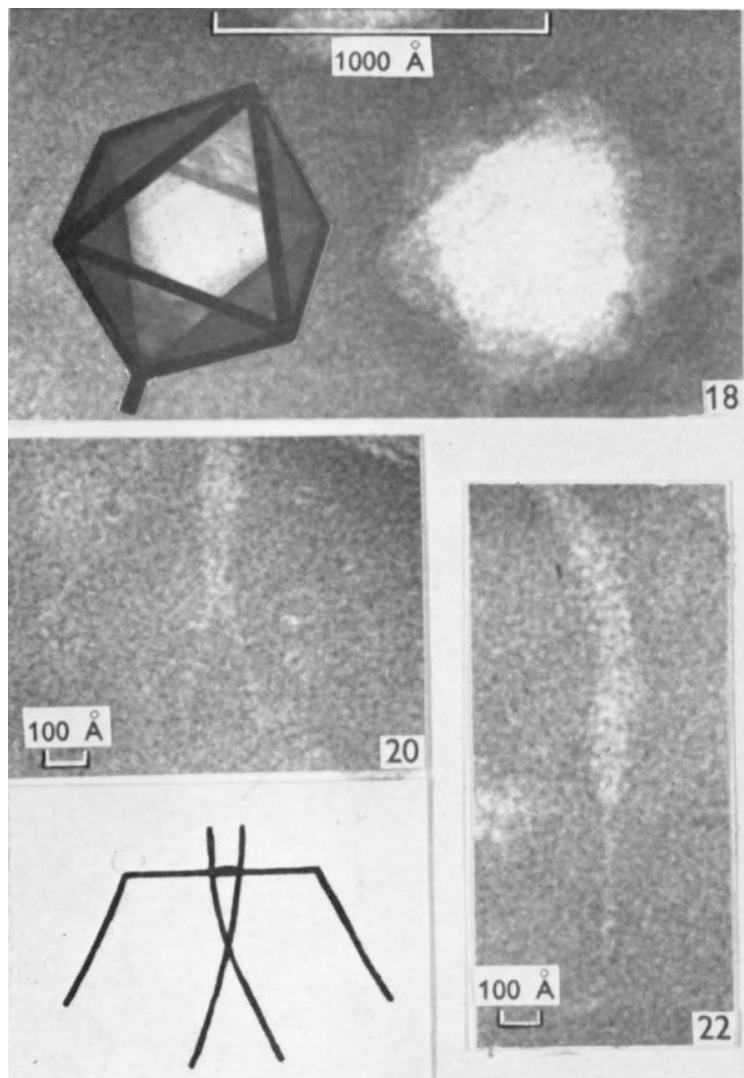

21
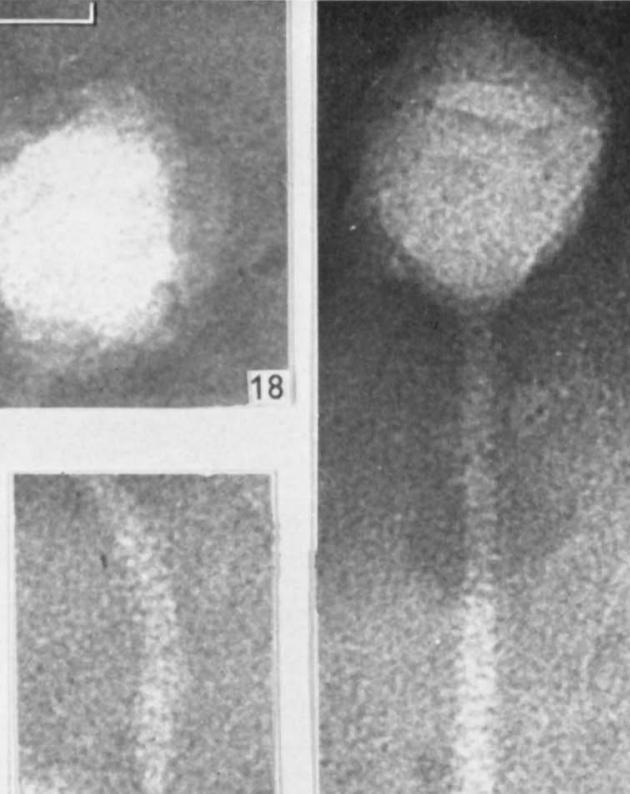

$100 \AA$

ᄂ
22

$100 \mathrm{~A}$
Plate 4
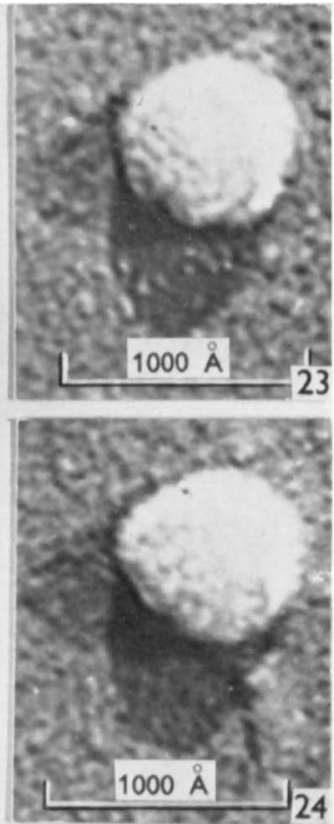

19

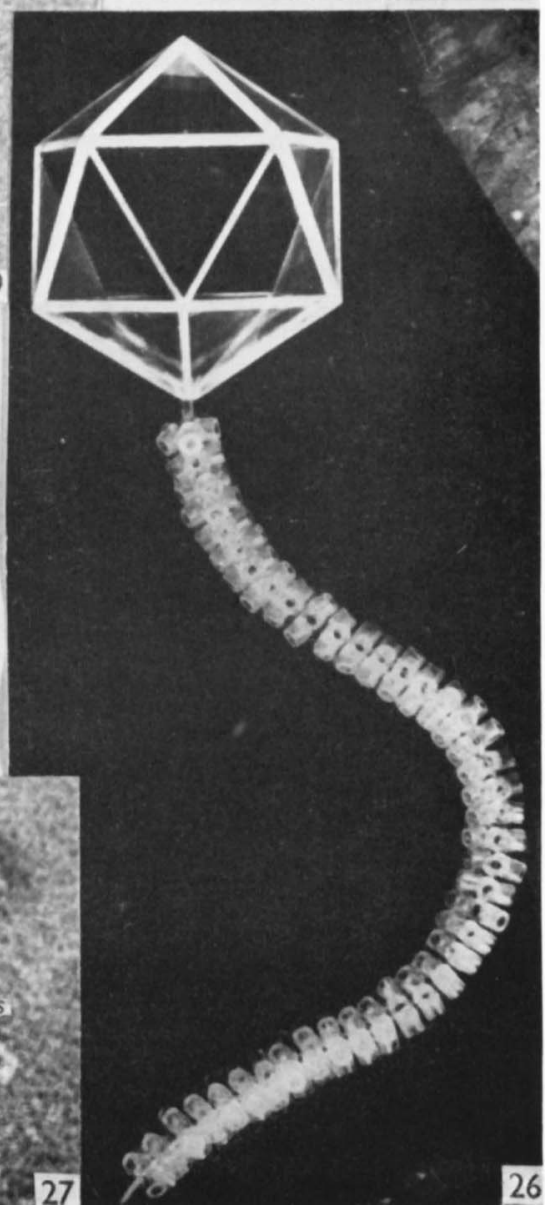

D. E. BRADLEY 
Journal of General Microbiology, Vol. 31, No. 3

Plate 5
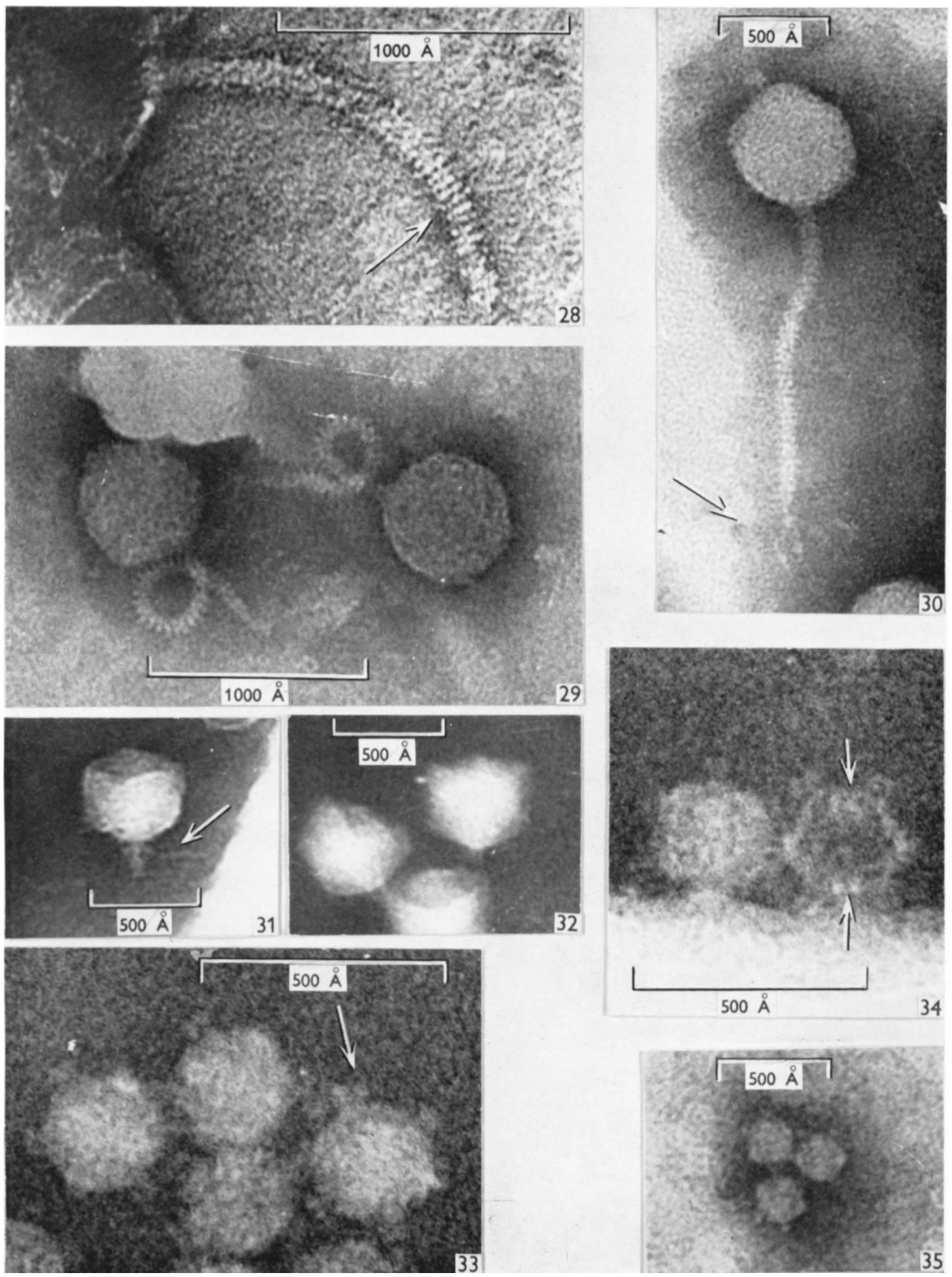

D. E. BRADLEY 

Plate 2

Figs. 7-9. Phage T2 in phosphotungstate, $\times \mathbf{3 3 3 , 0 0 0}$ (fig. 8 by courtesy of Academic Press Inc.). Fig. 10. Phage T2 in phosphotungstate, $\times 333,000 ; c$, core; $s$, contracted sheath; $f$, tail fibres (by courtesy of Academic Press Inc.).

\section{Plate 3}

Fig. 11. Extended sheaths, $\times 700,000$, of $a, \mathrm{~T} 2 ; b$, model; $c, \mathrm{~T} 4$; (by courtesy of Academic Press Inc.).

Figs. 12, 13. Phage $\mathrm{E} 1$ in phosphotungstate, $\times 333,000$.

Fig. 14. Head of phage $\phi 2, \times 250,000 ; a$, wire model of icosahedron; $b$, empty phage head in phosphotungstate; $c$, wire model of octahedron.

Fig. 15. Phage E1 with contracted sheath (phosphotungstate) $\times 333,000$.

Fig. 16. Phage E1 shadowed 2: 1 with platinum, $\times 100,000$.

Fig. 17. Contracted sheath of phage $66 \mathrm{t}^{-}, \times 333,000$.

\section{Plate 4}

Figs. 18, 19. Phage $\mathrm{Cl}$ in phosphotungstate, $\times 333,000$.

Fig. 20. Tail tip of phage $F 1, \times 333,000$.

Fig. 21. Drawing of tail tip of phage $F 1, \times 333,000$.

Fig. 22. Tail tip of phage $\mathrm{C} 1, \times 333,000$.

Figs. 23, 24. Heads of phage T5 platinum/carbon shadowed at $2: 1, \times 250,000$.

Fig. 25. Head of phage $T 5$ platinum/carbon shadowed at $2: 1, \times 450,000$.

Fig. 26. Model of phage T5.

Fig. 27. Phage T5 in uranyl acetate, $\times 375,000$.

\section{Plate 5}

Fig. 28. Phage T5 in uranyl acetate, $\times \mathbf{3 7 5 , 0 0 0}$.

Fig. 29. Phage $\beta 4$ in phosphotungstate, $\times 333,000$.

Fig. 30. Phage $\gamma 2$ in phosphotungstate, $\times 333,000$.

Figs. 31, 32. Phage T3 in phosphotungstate, $\times 333,000$.

Figs. 33, 34. Phage $\phi R$ in phosphotungstate, $\times 750,000$, fig. 34 by courtesy of Nature, Lond.

Fig. 35. Phage $\propto 3$ in phosphotungstate, $\times 333,000$, by courtesy of Nature, Lond. 